\title{
Synthesis and Properties of meso-Tetraphenylporphyrins with Sulfhydryl Groups
}

\author{
Kseniya A. Zhdanova, ${ }^{a}$ Artem V. Ezhov, ${ }^{a}$ Natal'ya A. Bragina $_{1}{ }^{a}$ \\ Nadezhda A. Aksenova, ${ }^{\mathrm{b}}$ Anna B. Solovieva, ${ }^{\mathrm{b}}$ Viktor N. Bagratashvili, ${ }^{\mathrm{c}}$ \\ Peter S. Timashev, ${ }^{\mathrm{c}}$ and Andrey F. Mironov ${ }^{\mathrm{a}}$
}
${ }^{a}$ M.V. Lomonosov Moscow State University of Fine Chemical Technologies, 119571 Moscow, Russian Federation ${ }^{\mathrm{b}}$ N.N. Semenov Institute of Chemical Physics, 119991 Moscow, Russian Federation
'Institute on Laser and Information Technologies, Russian Academy of Sciences, 142190 Troitsk, Moscow, Russian Federation
${ }^{\circledR}$ Corresponding author E-mail: n.bragina@mail.ru

\begin{abstract}
New thiol containing meso-arylporphyrins were synthesized and characterized by physico-chemical analysis. Optimal conditions of the reactions were selected. The singlet oxygen generation by thiolporphyrins at the conditions of model reaction of anthracene photooxidation was studied in chloroform solution.
\end{abstract}

Keywords: meso-Arylporphyrins, thiol containing porphyrins, quantum dots, synthesis, photooxidation.

\section{Синтез и свойства мезо-тетрафенилзамещенных порфиринов с сульфгидрильными группами}

\author{
К. А. Жданова, ${ }^{\text {a }}$ А. В. Ежов ${ }_{1}{ }^{a}$ Н. А. Брагина, ${ }^{a}$ Н. А. Аксенова, ${ }^{\text {b }}$ А. Б. Соловьева, \\ В. Н. Баграташвили, ${ }^{\text {}}$ П. С. Тимашев, ${ }^{\text {с } А . ~ Ф . ~ М и р о н о в ~}{ }^{a}$ \\ ${ }^{\mathrm{a}}$ Московский государственный университет тонких химических технологий им. М.В. Ломоносова, 119571 Москва, \\ Российская Федерация \\ ${ }^{\mathrm{b}}$ Институт химической физики им. Н.Н. Семёнова РАН, 119991 Москва, Российская Федерация \\ ${ }^{\mathrm{c}}$ Институт проблем лазерных и информационных технологий РАН, 142190 Троицк, Москва, Российская Федерация \\ ${ }^{\circledR}$ E-mail:n.bragina@mail.ru
}

\begin{abstract}
Новые амфифильные тиолсодержашие мезо-арилпорфирины синтезированы и охарактеризованы физикохимическими методами анализа. Подобраны оптимальные условия проведения реакций. Изучена генерация синглетного кислорода тиолпорфиринами в условиях модельной реакции фотоокисления антрацена.
\end{abstract}

Ключевые слова: мезо-Арилпорфирины, тиолпорфирины, квантовые точки, синтез, фотоокисление.

\section{Introduction}

Hybrid organo-inorganic or bioinorganic materials formation is novel, fast developing area of fundamental and applied researches. ${ }^{[1-3]}$ Due to the unique properties of these systems and their multifunctional nature they can be applied in various fields of science and technology from optics, microelectronics, to nanomedicine, etc. ${ }^{[4-5]}$ Porphyrin- quantum dots (QDs) hybrid materials can be applied as sensors, photosensitizers, diagnostic and therapeutic agents. ${ }^{[6-9]}$ In previous works ${ }^{[10-13]}$ the authors have reported on supramolecular assemblies based on QDs and porphyrins forming. However, such structures are often characterized by weak complexation constants, low values of energy transfer. In this regard covalent conjugates between QDs and dyes (e.g. porphyrins) could be more promising. Covalent bind- 
ing can be carried out by the interaction between the thiol groups introduced in the dye and the metal atoms on the surface of the quantum dot that has been shown at following works. ${ }^{[14,15]}$

It is known that the structure of porphyrin plays a key role in the formation of nanomaterials since it facilitates self-assembly and self-organization of molecules by noncovalent forces and increases the porphyrin solubility in organic media. ${ }^{[16]}$ Furthermore, the structure and physicochemical properties of porphyrins will determine the functional properties of the resulting conjugates. ${ }^{[17]}$ Despite the broad abilities that thiolcontaining porphyrins may open while forming the novel hybryd materials, the synthesis of these porphyrin is associated with certain difficulties. This work is focused on design of novel thiolcontaining tetraphenylporphyrins and study of their photosensitizing activity in model reaction of anthracene oxidation.

\section{Results and Discussion}

\section{Porphyrin Synthesis}

As long as thiolcontaining porphyrins with unprotected SH-groups can't be directly prepared by monopyrrole condensation $^{[18]}$ their synthesis can be carried out in two synthetic strategies: 1) modification of active functionnal groups (e.g. hydroxy- or amino-) at meso-positions of porphyrin precursors ${ }^{[}{ }^{[19]} 2$ ) using of benzaldehydes with protected thiol groups for further condensation with pyrrole followed by removing the protecting groups. ${ }^{[20,21]}$

Initially we have chosen the first strategy and synthesized monohydroxy substituted porphyrins which were further functionalized by dibromoalkanes. The target porphyrins 6-9 with terminal thiol groups were received by the conversion of bromoprecursors to the corresponding thioacetates and subsequent removing of the acetyl protection at last stage of reaction. However, this approach is characterized by low yields of the desired product, large number of stages and formation of by-products. Using the second strategy of synthesis based on the aldehyde monopyrrolic condensation with a benzaldehyde tioacetate derivative we have increased the thiol porphyrins yields (Scheme 1). The initial $p$-[(6-acetylthio)hexyloxy]benzaldehyde 1 was synthesized in two stages: $p$-hydroxybenzaldehyde was alkylated with 1,6-dibromohexane in $\mathrm{KOH}$ aqueous solution and then bromide was thioetherificated with potassium thioacetate. ${ }^{[20]}$ Benzaldehyde 1 was introduced into the mixed aldehyde monopyrrolic condensation by Lindsey method ${ }^{[22]}$ (Scheme 1). In this way previously described 5-(4-(6-ace-thylthiohexyloxy)phenyl)-10,15,20triphenylporphyrin 2 and the series of new thioacetate protected porphyrins 3-5, including porphyrin with three pentafluorophenyl groups were obtained. The products 3-5 were isolated by column chromatography on silica gel using an eluent mixture - dichloromethane:hexane, gradually increasing the polarity of the system. The protecting group was removed by the action of tetrabutylammonium cyanide $\left(\left(\mathrm{C}_{4} \mathrm{H}_{9}\right)_{4} \mathrm{~N}\right) \mathrm{CN}$ in an organic medium (chloroform:methanol 1:1) at $50{ }^{\circ} \mathrm{C}$ with the formation of thiols 6-9 according to the method. ${ }^{[19]}$ This method has proved to be more effective than removing of the protecting group under alkaline conditions.

The products 6-9 were isolated by column chromatography on silica gel collecting first running purple band. The structure and individuality of 1-6 were confirmed by TLC, UV-Vis and ${ }^{1} \mathrm{H},{ }^{13} \mathrm{C}$ NMR spectroscopy, MALDI-TOF mass-spectrometry. In ${ }^{1} \mathrm{H}$ NMR spectra the disappearance of acetate group at 2.33-2.37 ppm and appearance of low intensity peak of thiol group at $1.30-1.33$ ppm were observed. Figure 1 demonstrates the spectra of compound 3 with thioacetyl group and its corresponding thiol 7.

In MALDI-TOF mass-spectra of some products in addition to the peak of the molecular ion a peak $\left[\mathrm{M}^{2+}+\mathrm{CN}^{-}\right]^{+}$was observed. Probably, relatively stable complexes between the porphyrin and cyanide ion remaining in the reaction mixture are formed at conditions of the spectrum recording. Figure 1S (see Supplementary Information) shows the mass spectrum similar to that of compound $\mathbf{8}$.

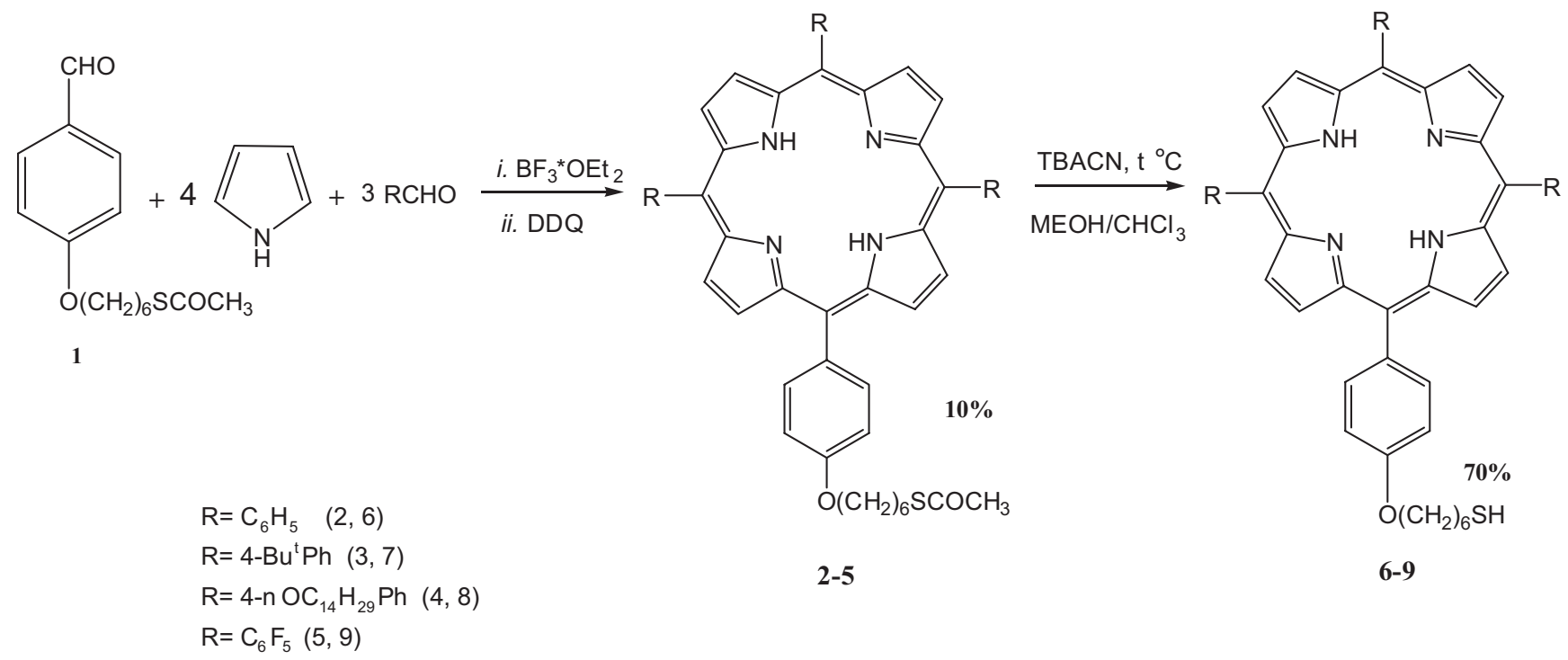

Scheme 1. $i-\mathrm{BF}_{3} \cdot \mathrm{OEt}_{2}, \mathrm{CH}_{2} \mathrm{Cl}_{2}, \mathrm{DDQ}, i i-\mathrm{TBACN}, \mathrm{CH}_{3} \mathrm{OH} / \mathrm{CH}_{2} \mathrm{Cl}_{2}, 50{ }^{\circ} \mathrm{C}$. 


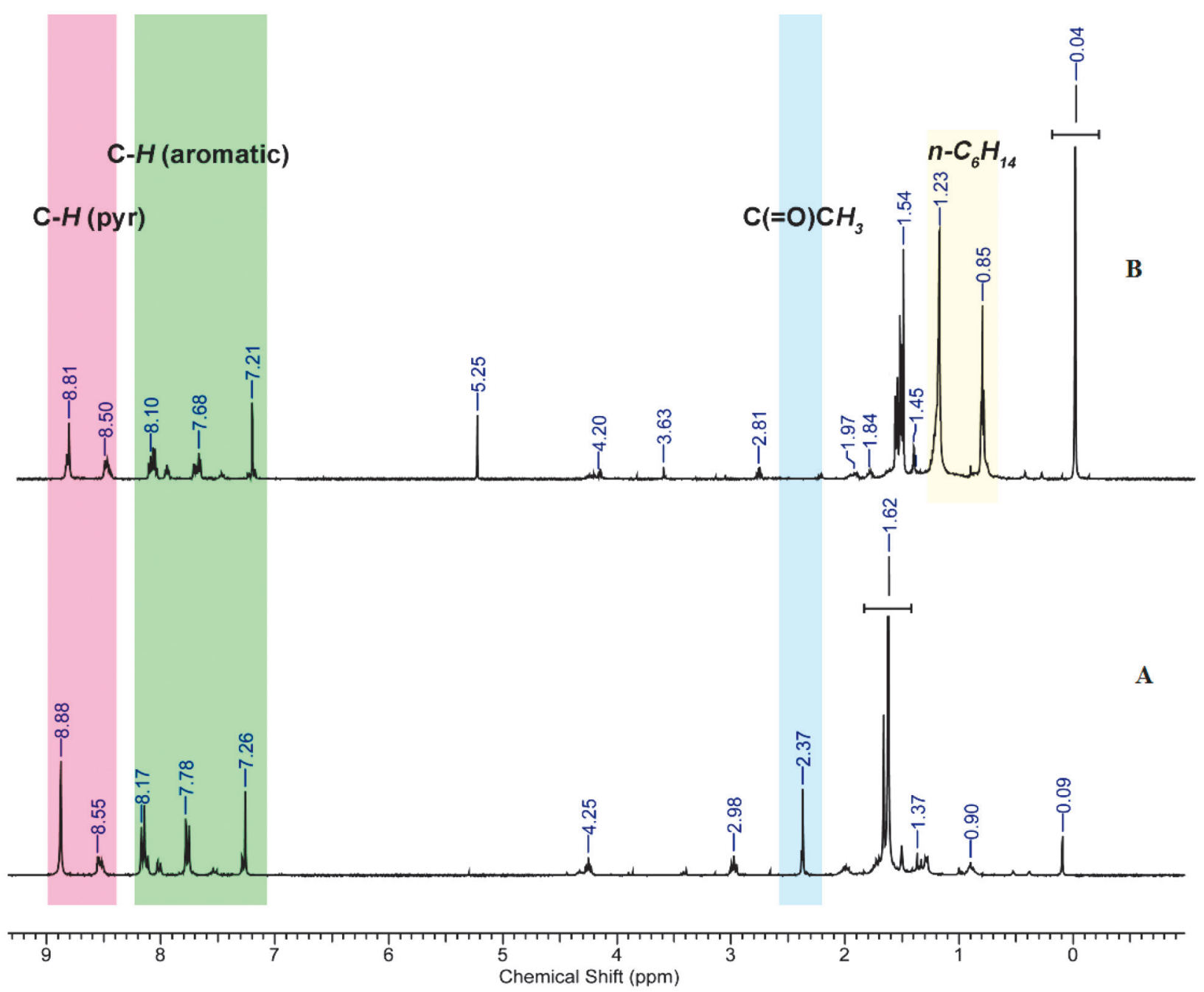

Figure 1. Comparison of ${ }^{1} \mathrm{H}$ NMR spectra of compounds 3 (A) and 7 (B).

\section{Spectral Characteristics and PPS Photosensitizing Activity}

The synthesized PPS were used as catalysts in the reaction of anthracene photooxidation. It was previously shown, ${ }^{[23]}$ that fluorinated tetraphenylporphyrins photosensitizing activity was higher than that of tetraphenylporphyrin in the oxidation reaction of anthracene in the organic phase. However, in our case the presence of fluorophenyl radical does not increase the effective constant of anthracene photooxidation.

Thus, the value of $k_{\text {eff }}$ of porphyrin 6 at $5 \cdot 10^{-6} \mathrm{M}$ concentration was $131 \mathrm{~mol} \cdot 1^{-1} \cdot \mathrm{s}^{-1}$, for porphyrin 7 at the same concentration - $130 \mathrm{~mol} \cdot \mathrm{l}^{-1} \cdot \mathrm{s}^{-1}$, for porphyrin 8 $132 \mathrm{~mol} \cdot \mathrm{l}^{-1} \cdot \mathrm{s}^{-1}$, for porphyrin $9-138 \mathrm{~mol} \cdot \mathrm{l}^{-1} \cdot \mathrm{s}^{-1}$. Probably, to increase the photosensitizing activity of porphyrin the symmetrical fluorosubstitution is required. Also, it was found that asymmetrically substituted tert-butyl and tetradecyloxygroups at the phenyl rings don't affect the activity of sulfurcontaining photosensitizing tetraphenylporphyrins. The study of the spectral properties of the synthesized porphyrins has shown that the intensity and position of absorption and fluorescence bands in the corresponding spectra are also the same (Figure 2S).

\section{Experimental}

To study the photosensitizing activity of porphyrins we have used a model reaction of photooxidation of anthracene in chloroform. ${ }^{[23]}$ Anthracene ("Reahim", Russia) was used as a substrate. Irradiation of the reaction mixture was carried out using phototherapy devices (APS "Polironik") $(\lambda=405 \mathrm{~nm}$, power $210 \mathrm{~mW})$. The reaction photooxidation mixture was stirred. The kinetics of the process have been monitored by changes of the anthracene concentration, which can be determined by the value of the optical density of the absorption band $(\lambda=360 \mathrm{~nm})$ in the UV spectrum of the anthracene. The rate constant was determined by the linear portion of the kinetic curve at the initial time. The effective rate constant of photooxidation of anthracene $(1 /(\mathrm{mol} \cdot \mathrm{s}))$ was calculated according to: $k_{\text {eff }}=\Delta c_{\mathrm{A}} /\left(c_{\mathrm{RN}} c_{\mathrm{A}} \Delta \mathrm{t}\right)$, where $c_{\mathrm{RN}}$ - the concentration of porphyrin photosensitizer (PPS), $c_{\mathrm{A}}$ and $\Delta c_{\mathrm{A}}$ - the concentration of the substrate and the variation of this concentration during $\Delta \mathrm{t}$, respectively.

All chemicals were obtained commercially and used as received unless otherwise noted. Pyrrole was purified by vacuum distillation; dichloromethane, hexane were dried by standard methods before using. Column chromatography was performed on silica gel G 60 (Merck Inc, 40-70 mesh). TLC was performed on pre-coated silica gel glass plates (silica gel 60, F-254, thickness $0.25 \mathrm{~mm}$ ) by Merck Inc. Benzaldehyde, 4-tert-butylbenzaldehyde, pentafluorobenzaldehyde, 1,6-dibromohexane, potassium thioacetate, tetrabutylammonium cyanide were purchased from Sigma- 
Aldrich and used without further purification. UV-vis spectra were recorded on TermoSpectronic Helios Alpha spectrophotometer in quartz cells of $0.5 \mathrm{~cm}$ thickness. All reported NMR results were obtained using Bruker 300 or $400 \mathrm{MHz}$ in $\mathrm{CD}_{3} \mathrm{CN}$. Mass-spectra were registered on "Ultraflex» (MALDI-TOF, matrix - DHB) equipment and ESI- MS Bruker MicroTOF-Q (Bruker Daltonics, Germany). Fluorescence spectra were recorded on Cary Eclipse (Agilent Technologies).

General procedure for the synthesis of porphyrins 2-5. Pyrrole $(0.1 \mathrm{~g}, 1.49 \mathrm{mmol})$, benzaldehyde $1(0.1 \mathrm{~g}, 0.37 \mathrm{mmol})$ and corresponding substituted benzaldehyde $(1.11 \mathrm{mmol})$ were dissolved in dichloromethane $(100 \mathrm{ml})$ under argon atmosphere. The reaction mixture was stirred for $15 \mathrm{~min}$, then trifluoroboron etherate $(16 \mu \mathrm{l}, 0.12 \mathrm{mmol})$ and absolute ethanol $(20 \mu \mathrm{l})$ were added, and the stirring was continued for $40 \mathrm{~min}$ at room temperature. Then DDQ $(0.16 \mathrm{~g}, 0.68 \mathrm{mmol})$ was added, and the stirring was continued for $1 \mathrm{~h}$ at room temperature. Finally, the products were separated by column chromatography on silica gel G60.

5-[4-(6-Thioacetoxyhexyl)phenyl]-10,15,20-triphenylporphyrin (2) was synthesized from pyrrole $(0.1 \mathrm{~g}, 1.49 \mathrm{mmol})$, benzaldehyde $1(0.1 \mathrm{~g}, 0.37 \mathrm{mmol})$ and benzaldehyde $(1.11 \mathrm{mmol}$, $0.119 \mathrm{~g})$. Yield $10.3 \%(30 \mathrm{mg})$. UV-Vis $\left(\mathrm{CH}_{2} \mathrm{Cl}_{2}\right) \lambda \mathrm{nm}: 419$, 516.5, 550, 590.5, 646 (band ratio 1:0.048:0.024:0.013:0.01). ${ }^{1} \mathrm{H}$ NMR $\left(\mathrm{CDCl}_{3}\right) \delta_{\mathrm{H}}$ ppm: $8.86(2 \mathrm{H}, \mathrm{d}, J=4.6 \mathrm{~Hz}, \mathrm{H} 3, \mathrm{H} 7), 8.80(6 \mathrm{H}$, br.s, H2, H8, H12, H13, H17, H18), 8.19 (6H, d, J=7.4 Hz, 2H), $8.08(2 \mathrm{H}, \mathrm{d}, J=8.4 \mathrm{~Hz}, 2 \mathrm{H}), 7.73-7.75(3 \mathrm{H}, \mathrm{m}, 4 \mathrm{H}), 7.68-7.64$ $(6 \mathrm{H}, \mathrm{m}, 3 \mathrm{H}), 7.21(2 \mathrm{H}, \mathrm{m}, 3 \mathrm{H}), 4.21\left(2 \mathrm{H}, \mathrm{m}, \mathrm{OCH}_{2} \mathrm{CH}_{2}\right), 2.93$ $\left(2 \mathrm{H}, \mathrm{m}, \mathrm{CH} \mathrm{H}_{2} \mathrm{SC}(\mathrm{O}) \mathrm{CH}_{3}\right), 2.33\left(3 \mathrm{H}, \mathrm{s}, \mathrm{SC}(\mathrm{O}) \mathrm{CH}_{3}\right), 2.02-1.92(2 \mathrm{H}$, $\left.\mathrm{m}, \mathrm{OCH}_{2} \mathrm{CH}_{2}\right), 1.71-1.51\left(6 \mathrm{H}, \mathrm{m},\left(\mathrm{CH}_{2}\right)_{3} \mathrm{CH}_{2} \mathrm{SC}(\mathrm{O}) \mathrm{CH}_{3}\right)$. Mass spectrum (MALDI-TOF) $m / z: 788.569\left[\mathrm{M}^{+}\right]$.

5-[4-(6-Thioacetoxyhexyl)phenyl]-10,15,20-tri(4-tertbutylphenyl)porphyrin (3) was synthesized from pyrrole (0.1 g, $1.49 \mathrm{mmol})$, benzaldehyde $1(0.1 \mathrm{~g}, 0.37 \mathrm{mmol})$ and 4-tertbutylbenzaldehyde (1.11 mmol, $0.180 \mathrm{~g}$ ). Yield $11.2 \%$ (39.7 mg). UV-Vis $\left(\mathrm{CH}_{2} \mathrm{Cl}_{2}\right) \lambda \mathrm{nm:}$ 421, 518, 553, 593, 650 (band ratio 1:0.036:0.021:0.009:0.011). ${ }^{1} \mathrm{H}$ NMR $\left(\mathrm{CDCl}_{3}\right) \delta_{\mathrm{H}} \mathrm{ppm}: 8.86(2 \mathrm{H}, \mathrm{d}$, $J=4.7 \mathrm{~Hz}, \mathrm{H} 3, \mathrm{H} 7), 8.82$ (6H, br.s, H2, H8, H12, H13, H17, H18), $8.19(6 \mathrm{H}, \mathrm{d}, J=7.2 \mathrm{~Hz}, 2 \mathrm{H}), 8.07(2 \mathrm{H}, \mathrm{d}, J=8.5 \mathrm{~Hz}, 2 \mathrm{H}), 7.74-7.68$ $(6 \mathrm{H}, \mathrm{m}, 3 \mathrm{H}), 7.21(2 \mathrm{H}, \mathrm{m}, 3 \mathrm{H}), 4.18\left(2 \mathrm{H}, \mathrm{t}, J=6.3 \mathrm{~Hz}, \mathrm{OCH}_{2} \mathrm{CH}_{2}\right)$, $2.80\left(2 \mathrm{H}, \mathrm{m}, \mathrm{CH}_{2} \mathrm{SC}(\mathrm{O}) \mathrm{CH}_{3}\right), 2.37\left(3 \mathrm{H}, \mathrm{s}, \mathrm{CH}_{2} \mathrm{SC}(\mathrm{O}) \mathrm{CH}_{3}\right) 1.96$ $\left(2 \mathrm{H}, \mathrm{m}, \mathrm{OCH}_{2} \mathrm{CH}_{2}\right), 1.83\left(2 \mathrm{H}, \mathrm{m}, J=7.3 \mathrm{~Hz}, \mathrm{OCH}_{2} \mathrm{CH}_{2} \mathrm{CH}_{2} \mathrm{CH}_{2}\right)$, $1.70-1.62\left(6 \mathrm{H}, \mathrm{m},\left(\mathrm{CH}_{2}\right)_{3} \mathrm{CH}_{2} \mathrm{SC}(\mathrm{O}) \mathrm{CH}_{3}\right), 1.60\left(27 \mathrm{H}, \mathrm{s},{ }^{2} \mathrm{Bu}\right)$. Mass spectrum (MALDI-TOF) $m / z: 957.569[\mathrm{MH}]^{+}$.

5-[4-(6-Thioacetoxyhexyl)phenyl]-10,15,20-tri(4tetradecyloxyphenyl)porphyrin (4) was synthesized from pyrrole (0.1 g, $1.49 \mathrm{mmol})$, benzaldehyde $1(0.1 \mathrm{~g}, 0.37 \mathrm{mmol})$ and 4- tetradecyloxybenzaldehyde ( $1.11 \mathrm{mmol}, 0.353 \mathrm{~g})$. Yield $12.5 \%$ (66 mg). UV-Vis $\left(\mathrm{CH}_{2} \mathrm{Cl}_{2}\right) \lambda \mathrm{nm}: 423,519,555,595,650$ (band ratio 1:0.033:0.023:0.009:0.012). ${ }^{1} \mathrm{HNMR}\left(\mathrm{CDCl}_{3}\right) \delta_{\mathrm{H}}$ ppm: $8.85(8 \mathrm{H}$, br.s, H2, H3, H7, H8, H12, H13, H17, H18), 8.08 (8H, d, J=8.2 Hz, 2H), $7.24-7.21(8 \mathrm{H}, \mathrm{m}, 3 \mathrm{H}), 4.20-4.16\left(8 \mathrm{H}, \mathrm{m}, \mathrm{OCH}_{2} \mathrm{CH}_{2}\right), 2.94(2 \mathrm{H}, \mathrm{t}$, $\left.J=7.3 \mathrm{~Hz}, \mathrm{CH}_{2} \mathrm{SC}(\mathrm{O}) \mathrm{CH}_{3}\right), 2.34\left(3 \mathrm{H}, \mathrm{s}, \mathrm{CH}_{2} \mathrm{SC}(\mathrm{O}) \mathrm{CH}_{3}\right), 1.97-1.92$ $\left(8 \mathrm{H}, \mathrm{m}, \mathrm{OCH}_{2} \mathrm{CH}_{2}\right), 1.77-1.70\left(2 \mathrm{H}, \mathrm{m}, \mathrm{CH}_{2} \mathrm{CH}_{2} \mathrm{SC}(\mathrm{O}) \mathrm{CH}_{3}\right), 1.71-$ $1.67\left(6 \mathrm{H}, \mathrm{m}, \mathrm{O}\left(\mathrm{CH}_{2}\right)_{2} \mathrm{CH}_{2}\right), 1.64-1.51\left(4 \mathrm{H}, \mathrm{m},\left(\mathrm{CH}_{2}\right)_{2}\left(\mathrm{CH}_{2}\right)_{2} \mathrm{SC}(\mathrm{O})\right.$ $\left.\mathrm{CH}_{3}\right), 1.49-1.23\left(60 \mathrm{H}, \mathrm{m},\left(\mathrm{CH}_{2}\right)_{10} \mathrm{CH}_{3}\right), 0.89(9 \mathrm{H}, \mathrm{t}, J=6.2 \mathrm{~Hz}$, $\mathrm{CH}_{2} \mathrm{CH}_{3}$ ). Mass spectrum (MALDI-TOF) $\mathrm{m} / z: 1427.882[\mathrm{M}+2 \mathrm{H}]^{+}$.

5-[4-(6-Thioacetoxyhexyl)phenyl]-10,15,20-tri(pentafluorophenyl)porphyrin 5 was synthesized from pyrrole $(0.1 \mathrm{~g}, 1.49 \mathrm{mmol})$, benzaldehyde $1(0.1 \mathrm{~g}, 0.37 \mathrm{mmol})$ and pentafluorobenzaldehyde $(1.11 \mathrm{mmol}, 0.218 \mathrm{~g})$. Yield $9 \%(33.8 \mathrm{mg})$. UV-Vis $\left(\mathrm{CH}_{2} \mathrm{Cl}_{2}\right) \lambda$ nm: 418, 509, 545, 579 (band ratio 1:0.029:0.058:0.019). ${ }^{1} \mathrm{H}$ NMR $\left(\mathrm{CDCl}_{3}\right) \delta_{\mathrm{H}}$ ppm: $8.99(2 \mathrm{H}, \mathrm{d}, J=4.6 \mathrm{~Hz}, \mathrm{H} 3, \mathrm{H} 7), 8.86(4 \mathrm{H}, \mathrm{m}$, $\mathrm{H} 2, \mathrm{H} 8, \mathrm{H} 12, \mathrm{H} 18), 8.79$ (2H, d, J=4.4 Hz, H13, H17), 8.07 (2H, d, $J=8.5 \mathrm{~Hz}, 2 \mathrm{H}), 7.21(2 \mathrm{H}, \mathrm{sc}, 3 \mathrm{H}), 4.21(2 \mathrm{H}, \mathrm{t}, J=6.41 \mathrm{~Hz}$, $\left.\mathrm{OCH}_{2} \mathrm{CH}_{2}\right), 2.93\left(2 \mathrm{H}, \mathrm{t}, J=7.32 \mathrm{~Hz} \mathrm{CH}_{2} \mathrm{SC}(\mathrm{O}) \mathrm{CH}_{3}\right), 2.33(3 \mathrm{H}, \mathrm{s}$, $\left.\mathrm{CH}_{2} \mathrm{SC}(\mathrm{O}) \mathrm{CH}_{3}\right), 1.93-1.98\left(2 \mathrm{H}, \mathrm{m}, \mathrm{OCH}_{2} \mathrm{CH}_{2}\right), 1.70-1.50(6 \mathrm{H}$, $\left.\mathrm{m},\left(\mathrm{CH}_{2}\right)_{3} \mathrm{CH}_{2} \mathrm{SC}(\mathrm{O}) \mathrm{CH}_{3}\right)$. Mass spectrum (MALDI-TOF) $\mathrm{m} / \mathrm{z}$ : $1060.067[\mathrm{M}+2 \mathrm{H}]^{+}$.
General procedure for the synthesis of thiolporphyrins 6-9. Thioacetyl-protected porphyrin $(0.1 \mathrm{mmol})$ was dissolved in $\mathrm{CH}_{2} \mathrm{Cl}_{2}(5 \mathrm{ml})$ under argon atmosphere at room temperature, then tetrabutylammonium cyanide $(0.3 \mathrm{mmol}, 0.081 \mathrm{mg})$ in methanol (4 ml) was added. The resulting mixture was heated up to $50^{\circ} \mathrm{C}$ and stirred for $10 \mathrm{~h}$. Reaction mixture was transferred into a separatory funnel, organic phase was extracted with ammonium chloride, dried over $\mathrm{Na}_{2} \mathrm{SO}_{4}$ and evaporated. Finally, the products were separated by column chromatography on silica gel G60.

5-[4-(6-Thiohexyloxy)phenyl]-10,15,20-triphenylporphyrin (6) was synthesized from porphyrin $2(0.1 \mathrm{mmol}, 79 \mathrm{mg})$. Yield $73 \%(54.4 \mathrm{mg})$. UV-Vis $\left(\mathrm{CH}_{2} \mathrm{Cl}_{2}\right) \lambda \mathrm{nm}: 419,516,550,590,647$ (band ratio 1:0.037:0.017:0.009:0.009). ${ }^{1} \mathrm{H} \mathrm{NMR}\left(\mathrm{CDCl}_{3}\right) \delta_{\mathrm{H}} \mathrm{ppm}$ : $8.86(2 \mathrm{H}, \mathrm{d}, J=4.7 \mathrm{~Hz}, \mathrm{H} 3, \mathrm{H} 7), 8.82$ (6H, br.s, H2, H8, H12, H13, H17, H18), $8.19(6 \mathrm{H}, \mathrm{d}, J=7.2 \mathrm{~Hz}, 2 \mathrm{H}), 8.07(2 \mathrm{H}, \mathrm{d}, J=8.5 \mathrm{~Hz}$, 2H), 7.74-7.71 (3H, m, 4H), 7.70-7.68 (6H, m, 3H), $7.21(2 \mathrm{H}, \mathrm{m}$, $3 \mathrm{H}), 4.18\left(2 \mathrm{H}, \mathrm{t}, J=6.3 \mathrm{~Hz}, \mathrm{OCH}_{2} \mathrm{CH}_{2}\right), 2.80(2 \mathrm{H}, \mathrm{m}, \mathrm{CH}, \mathrm{SH}), 1.96$ $\left(2 \mathrm{H}, \mathrm{m}, \mathrm{OCH}_{2} \mathrm{CH}_{2}\right), 1.83\left(2 \mathrm{H}, \mathrm{q}, J=7.3 \mathrm{~Hz}, \mathrm{OCH}_{2} \mathrm{CH}_{2} \mathrm{CH}_{2} \mathrm{CH}_{2}\right)$, $1.67-1.59\left(4 \mathrm{H}, \mathrm{m},\left(\mathrm{CH}_{2}\right)_{2} \mathrm{SH}\right), 1.44(1 \mathrm{H}, \mathrm{s}, \mathrm{SH}),-2.74(2 \mathrm{H}$, br.s, $\mathrm{NH})$. Mass spectrum (MALDI-TOF) $\mathrm{m} / \mathrm{z}$ : $747.299[\mathrm{M}+\mathrm{H}]^{+}$.

5-[4-(6-Thiohexyloxy)phenyl]-10,15,20-tri(4-tert-butylphenyl)porphyrin (7) was synthesized from porphyrin $3(0.1 \mathrm{mmol}$, $95.7 \mathrm{mg})$. Yield $72 \%(66 \mathrm{mg})$. UV-Vis $\left(\mathrm{CH}_{2} \mathrm{Cl}_{2}\right) \lambda \mathrm{nm}: 421,518$, 554, 592, 650 (band ratio 1:0.036:0.021:0.009:0.012). ${ }^{1} \mathrm{H}$ NMR $\left.\left(\mathrm{CDCl}_{3}\right) \delta_{\mathrm{H}} \mathrm{ppm}\right): 8.83(2 \mathrm{H}, \mathrm{d}, J=4.7 \mathrm{~Hz}, \mathrm{H} 3, \mathrm{H} 7), 8.81(6 \mathrm{H}$, br.s, $\mathrm{H} 2, \mathrm{H} 8, \mathrm{H} 12, \mathrm{H} 13, \mathrm{H} 17, \mathrm{H} 18), 8.12(6 \mathrm{H}, \mathrm{d}, J=7.2 \mathrm{~Hz}, 2 \mathrm{H}), 8.06$ $(2 \mathrm{H}, \mathrm{d}, J=8.5 \mathrm{~Hz}, 2 \mathrm{H}), 7.73-7.66(6 \mathrm{H}, \mathrm{m}, 3 \mathrm{H}), 7.21(2 \mathrm{H}, \mathrm{m}, 3 \mathrm{H})$, $4.18\left(2 \mathrm{H}, \mathrm{t}, J=6.3 \mathrm{~Hz}, \mathrm{OCH}_{2} \mathrm{CH}_{2}\right), 2.80(2 \mathrm{H}, \mathrm{m}, \mathrm{CH}, \mathrm{SH}), 1.96$ $\left(2 \mathrm{H}, \mathrm{m}, \mathrm{OCH}_{2} \mathrm{CH}_{2}\right), 1.83\left(2 \mathrm{H}, \mathrm{m}, J=7.3 \mathrm{~Hz}, \mathrm{OCH}_{2} \mathrm{CH}_{2} \mathrm{CH}_{2} \mathrm{CH}_{2}\right)$, 1.67-1.59 (4H, m, $\left.\left(\mathrm{CH}_{2}\right)_{3} \mathrm{CH}_{2} \mathrm{SH}\right), 1.57\left(27 \mathrm{H}, \mathrm{s},{ }^{t} \mathrm{Bu}\right), 1.30(1 \mathrm{H}, \mathrm{s}$, $\mathrm{SH}$ ). Mass spectrum (MALDI-TOF) $m / z: 915.499[\mathrm{MH}]^{+}$.

5-[4-(6-Thiohexyloxy)phenyl]-10,15,20-tri(4-tetradecyloxyphenyl)porphyrin (8) was synthesized from porphyrin $4(0.1 \mathrm{mmol}$, $138 \mathrm{mg})$. Yield $77 \%$ (106 mg). UV-Vis $\left(\mathrm{CH}_{2} \mathrm{Cl}_{2}\right) \lambda \mathrm{nm}: 423,519$, 556, 593, 653 (band ratio 1:0.036:0.02:0.012:0.009). ${ }^{1} \mathrm{H}$ NMR $\left(\mathrm{CDCl}_{3}\right) \delta_{\mathrm{H}}$ ppm: $8.83(8 \mathrm{H}$, br.s, H2, H3, H7, H8, H12, H13, H17, $\mathrm{H} 18), 8.08$ (8H, m, 2H), 7.27-7.33 (8H, m, 3H), 4.22-4.15 (8H, $\left.\mathrm{m}, \mathrm{OCH}_{2} \mathrm{CH}_{2}\right), 2.98\left(2 \mathrm{H}, \mathrm{t}, J=7.26 \mathrm{~Hz}, \mathrm{CH}_{2} \mathrm{SH}\right), 1.95-1.91(8 \mathrm{H}$, $\left.\mathrm{m}, \mathrm{OCH}_{2} \mathrm{CH}_{2}\right), 1.77-1.70\left(2 \mathrm{H}, \mathrm{m}, \mathrm{CH}_{2} \mathrm{CH}_{2} \mathrm{SH}\right), 1.71-1.67(6 \mathrm{H}, \mathrm{m}$, $\left.\mathrm{O}\left(\mathrm{CH}_{2}\right)_{2} \mathrm{CH}\right)_{2}, 1.64-1.51\left(4 \mathrm{H}, \mathrm{m},\left(\mathrm{CH}_{2}\right)_{2}\left(\mathrm{CH}_{2}\right)_{2} \mathrm{SH}\right), 1.47-1.23(60 \mathrm{H}$, m, $\left.\left(\mathrm{CH}_{2}\right)_{10} \mathrm{CH}_{3}\right), 0.89\left(9 \mathrm{H}, \mathrm{t}, J=6.2 \mathrm{~Hz}, \mathrm{CH}_{2} \mathrm{CH}_{3}\right)$. Mass spectrum (MALDI-TOF) $m / z$ : for $[\mathrm{M}+2 \mathrm{H}]^{+}\left(\mathrm{C}_{94} \mathrm{H}_{130} \mathrm{~N}_{4} \mathrm{O}_{5} \mathrm{~S}\right)$ found: 1427.882 , calcd.: 1427.980. MS (MALDI-TOF) $m / z$ : $1409.878\left[\mathrm{M}^{2+}+\mathrm{CN}^{-}\right]$

5-[4-(6-Thiohexyloxy)phenyl]-10,15,20-tri(pentafluorophenyl)porphyrin 9 was synthesized from porphyrin $5(0.1 \mathrm{mmol}$, $102 \mathrm{mg}$ ). Yield - $74 \%(75 \mathrm{mg})$. UV-Vis $\left(\mathrm{CH}_{2} \mathrm{Cl}_{2}\right) \lambda$ (band ratio)/ nm: 418, 510, 546, 581 (1:0.031:0.062:0.02). ${ }^{2} \mathrm{H} \mathrm{NMR}\left(\mathrm{CDCl}_{3}\right) \delta_{\mathrm{H}}$ ppm: $8.86(2 \mathrm{H}, \mathrm{d}, J=4.6 \mathrm{~Hz}, \mathrm{H} 3, \mathrm{H} 7), 8.67$ (4H, m, H2, H8, H12, H18), 8.49 (2H, d, $J=4.4 \mathrm{~Hz}, \mathrm{H} 13, \mathrm{H} 17), 8.08(2 \mathrm{H}, \mathrm{d}, J=8.5 \mathrm{~Hz}$, $2 \mathrm{H}), 7.21(2 \mathrm{H}, \mathrm{m}, 3 \mathrm{H}), 4.19\left(2 \mathrm{H}, \mathrm{t}, J=6.44 \mathrm{~Hz}, \mathrm{OCH}_{2} \mathrm{CH}_{2}\right), 2.93$ $\left(2 \mathrm{H}, \mathrm{t}, J=7.32 \mathrm{~Hz}, \mathrm{CH}, \mathrm{SC}(\mathrm{O}) \mathrm{CH}_{3}\right), 1.93-1.98\left(2 \mathrm{H}, \mathrm{m}, \mathrm{OCH}_{2} \mathrm{CH}_{2}\right)$, $1.70-1.50\left(6 \mathrm{H}, \mathrm{m},\left(\mathrm{CH}_{2}\right)_{3} \mathrm{CH}_{2} \mathrm{SH}\right), 1.33(1 \mathrm{H}, \mathrm{s}, \mathrm{SH})$. Mass spectrum (MALDI-TOF) $m / z: 1017.098[\mathrm{MH}]^{+}$.

\section{Conclusions}

In summary, novel asymmetrical thiolcontaining porphyrins design strategy with high yields has been developed. The porphyrin structure was confirmed by UV-Vis, and ${ }^{1} \mathrm{H}$, ${ }^{13} \mathrm{C}$ NMR spectroscopy, MALDI-TOF mass-spectrometry. The photosensitizing activity of the porphyrins was studied.

Acknowledgements. The authors thank Russian Scientific Foundation Grant no. 14-13-01422 (porphyrin synthesis and its characterization) and Russian Foundation of Basic Research 13-03-12046 ofi_m (photosensitizing activity experiments). 


\section{References}

1. Sanchez C., Belleville P., Popall M., Nicole L. Chem. Soc. Rev. 2011, 40, 696-753.

2. Wang H., Dai H. Chem. Soc. Rev. 2013, 42, 3088-3113.

3. Biju V. Chem. Soc. Rev. 2014, 43, 744-764.

4. Umeyama T., Imahori H. J. Phys. Chem. C 2013, 117, 3195-3209.

5. Sanchez C., Boissiere C., Cassaignon S., Chaneac C., Durupthy O., Faustini M., Grosso D., Laberty-Robert C., Nicole L., Portehault D., Ribot F., Rozes L., Sassoye C. Chem. Mater. 2014, 26, 221-238.

6. Pagona G., Zervaki G.E., Sandanayaka A.S.D., Ito O., Charalambidis G., Hasobe T., Coutsolelos A.G., Tagmatarchis N. J. Phys. Chem. C 2012, 116, 9439-9449.

7. Carvalho C.M.B., Alves E., Costa L., Tomé J.P.C., Faustino M.A.F., Neves M., Tomé A. C., Cavaleiro J.A.S., Almeida A., Cunha A., Lin Z., Rocha J. ACS Nano 2010, 4, 7133-7140.

8. Said A.J., Poize G., Martini C., Ferry D., Marine W., Giorgio S., Fages F., Hocq J., Boucle J., Nelson J., Durrant J.R., Ackermann J. J. Phys. Chem. C 2010, 114, 11273-11278.

9. Sortino S. J. Mater. Chem. 2012, 22, 301-318.

10. Mandal S., Rahaman M., Sadhu S., Nayak S.K., Patra A. J. Phys. Chem. C 2013, 117, 3069-3077.

11. Borissevitch I.E., Parra G.G., Zagidullin V.E., Lukashev E.P., Knox P.P., Paschenko V.Z., Rubin A.B. J. Luminescence 2013, $134,83-87$.
12. Zhdanova K.A., Bragina N.A., Bagratashvili V.N., Timashev P.S., Mironov A.F. Mendeleev Commun. 2014, 24, 247-249.

13. Zenkevich E.I., Blaudeck T., Milekhin A., von Borczyskowski C. Int. J. Spectr. 2012, Article ID 971791.

14. Imahori H., Kashiwagi Y., Endo Y., Hanada T., Nishimura Y., Yamazaki I., Araki Y., Ito O., Fukuzumi S. Langmuir 2004, 20, 73-81.

15. Zak J., Yuan H., Ho M., Woo L.K., Porter M.D. Langmuir 1993, 9, 2772-2774.

16. Drain C.M., Varotto A., Radivojevic I. Chem. Rev. 2009, 109, 1630-1658.

17. Zenkevich E.I., Borczyskowski C. Macroheterocycles 2009, 2, 206-220.

18. Adler A.D., Longo E.R., Shergalis W. J. Am. Chem. Soc. 1964, 86, 3145-3149.

19. Xue C., Xu Y., Pang Y., Yu D., Dai L., Gao M., Urbas A., Li Q. Langmuir 2012, 28, 5956-5963.

20. Nishimura N., Ooi M., Shimazu K., Fujii H., Uosaki K. J. Electroanal. Chem. 1999, 473, 75-84.

21. Piotrowski P., Pawłowska J., Pawłowski J., Wieckowska A., Bilewicza R., Kaim A. J. Mater. Chem. A. 2014, 2, 2353-2362.

22. Lindsey J.S., Schreiman I.C., Hsu H.C., Kearney P.C., Marguerettaz A.M. J. Org. Chem. 1987, 52, 827-836.

23. Shershnev I.V., Glagolev N.N., Bragina N.A., Timashev P.S., Bagratashvili V.N., Solovieva A.B. Russ. J. Phys. Chem. B 2014, 8, 1095-1099. 